Comment. Math. Helv. 79 (2004) 451-470

0010-2571/04/030451-20

DOI $10.1007 / \mathrm{s} 00014-003-0792-7$
(C) 2004 Birkhäuser Verlag, Basel

Commentarii Mathematici Helvetici

\title{
Estimates on the growth of meromorphic solutions of linear differential equations
}

Yik-Man Chiang and Walter K. Hayman

Dedicated to the memory of Steven Bank

Abstract. We give a pointwise estimate of meromorphic solutions of linear differential equations with coefficients meromorphic in a finite disk or in the open plane. Our results improve some earlier estimates of Bank and Laine. In particular we show that the growth of meromorphic solutions with $\delta(\infty)>0$ can be estimated in terms of initial conditions of the solution at or near the origin and the characteristic functions of the coefficients. Examples show that the estimates are sharp in a certain sense. Our results give an affirmative answer to a question of Milne Anderson.

Our method consists of two steps. In Theorem 2.1 we construct a path $\Gamma\left(\theta_{0}, \rho, t\right)$ consisting of the ray

$$
z=\tau e^{i \theta_{0}}, \quad \rho \leq \tau \leq t,
$$

followed by the circle

$$
z=t e^{i \theta}, \quad \theta_{0} \leq \theta \leq \theta_{0}+2 \pi,
$$

on which the coefficients are all bounded in terms of the sum of their characteristic functions on a larger circle. In Theorem 2.2 we show how such an estimate for the coefficients leads to a corresponding bound for the solution on $|z|=t$. Putting these two steps together we obtain our main result, Theorem 2.3 .

Mathematics Subject Classification (2000). 34M10, 34M05.

Keywords. Linear differential equations, growth of meromorphic solutions, Nevanlinna characteristics.

The research of the first author was partially supported by the University Grants Council of the Hong Kong Special Administrative Region, China (HKUST711/96P). 


\section{Background and earlier results}

We consider meromorphic solutions of the differential equation

$$
y^{(n)}(z)+\sum_{\nu=0}^{n-1} f_{\nu}(z) y^{(\nu)}(z)=f_{n}(z),
$$

where the $f_{\nu}(z)$ are meromorphic in a disk $|z| \leq R$. We assume that the reader is familiar with the fundamental concepts of Nevanlinna Theory. However for the purpose of this paper we ignore poles at the origin. Thus if $f(0) \neq \infty$ our notation is standard (see [11, Chapter 1]), however if $f(0)=\infty, n(t)$ denotes the number of poles of $f$ in $0<|z| \leq t$ and $N(t), \bar{N}(t)$ are derived in the usual way.

Bank and Laine [6, Theorem 3] have shown that the Nevanlinna characteristic $T(r, y)$ of every meromorphic solution $y(z)$ of

$$
y^{(n)}(z)+\sum_{\nu=0}^{n-1} f_{\nu}(z) y^{(\nu)}(z)=0
$$

can be estimated in terms of the poles and the distinct zeros of $y$ and the characteristic functions of the coefficients. Here as in the (1.1), the coefficients are meromorphic. More precisely they proved

Theorem 1.1. Suppose that the coefficients of (1.2) are arbitrary meromorphic functions and that $y(z)$ is a meromorphic solution of (1.1). If

$$
\Phi(r)=\max _{0 \leq i \leq n}\left(\log r, T\left(r, f_{i}\right)\right),
$$

then for any $\sigma, \sigma>1$, there exist positive constants $c, c_{1}$ and $r_{0}$, such that for $r \geq r_{0}$,

$$
T(r, y) \leq c\left\{r N(\sigma r, y)+r^{2} \exp \left(c_{1} J(\sigma r) \log (r J(\sigma r))\right\},\right.
$$

where

$$
J(r)=\bar{N}(r, 1 / y)+\Phi(r) .
$$

We refer to [4], Laine [12] and the references therein for the literature on the growth of entire solutions of (1.1). The results go back to Valiron and Wittich, and it suffices to mention that we can find an upper bound of the Nevanlinna characteristic of the entire solution of (1.1) in terms of the Nevanlinna characteristics of the coefficients. We also mention the works of Frei [7], Strelitz [14] and Gundersen, Steinbart and Wang [8] in this connection.

Suppose now that (1.2) has rational coefficients and that it admits a meromorphic solution. Then the solution can have at most a finite number of poles because they can only appear at the poles of the coefficients. It follows that the growth of the characteristic function of the meromorphic solutions is very much like that of entire solutions and so they can be estimated in terms of the characteristic of the 
coefficients by the methods of Valiron and Wittich. However, getting a precise upper bound for a meromorphic solution becomes a more difficult task, since many tools, such as the Wiman-Valiron theory, are only available for entire function solutions.

It is natural to ask whether one can obtain an upper bound for $T(r, y)=$ $m(r, y)+N(r, y)$ in terms of the characteristics $T\left(r, f_{\nu}\right)$ of the coefficients alone. An example of Bank [5] (see also [1]) shows that this is in general impossible. He proved that the characteristics $T(r, y)$ of the meromorphic solution of the equation

$$
y^{\prime}(z)=f_{0}(z) y(z)
$$

can grow arbitrarily fast, while the meromorphic coefficient $f_{0}(z)$ grows arbitrarily slowly. Rather surprisingly we are able to show in our main result Theorem 2.3 that it is possible to obtain a pointwise estimate for $y(z)$ and hence a bound for $m(r, y)$ in terms of the characteristics of the $f_{\nu}$.

The poles of $y$ can only occur at the poles of $f_{\nu}$, so that

$$
\bar{N}(r, y) \leq \sum_{\nu=0}^{n} \bar{N}\left(r, f_{\nu}\right) \leq \sum_{\nu=0}^{n} T\left(r, f_{\nu}\right)
$$

Thus for $T(r, y)=m(r, y)+N(r, y)$ to be large, the average multiplicity

$$
N(r, y) / \bar{N}(r, y)
$$

must be extremely large. The gist of Bank's construction of his examples is that his meromorphic solution of (1.5) has $\delta(\infty)=0$ [5, Lemma]. This feature of the Bank examples is thus essential not only for the solutions of (1.5) but also for those of (1.1).

In the next section we state our results in detail and deduce Theorem 2.3 from Theorems 2.1 and 2.2. In Section 3 we prove Theorem 2.2 which is quite simple. The proof of Theorem 2.1 is completed in Section 4. In Section 5 we shall briefly consider the analogous problem for equation (1.1) in the unit disk. In Section 6 we obtain estimates for $m(r, y)$ in terms of $T(r)=\sum T\left(r, f_{\nu}\right)$ outside an exceptional set of $r$ having finite measure or finite logarithmic measure. We note that the quantities $A, R, K, K_{1}, K_{2}, \cdots$ which appear in the rest of this paper are not necessarily the same each time they occur. 


\section{Statements of results}

We prove the following

Theorem 2.1. Suppose that the functions $f_{\nu}(z), \nu=0, \cdots, n-1$ are meromorphic in $|z| \leq R$ and that $0<\rho<r<R<+\infty$. Then there exists $t$ such that $r<t<\frac{1}{4}(3 r+R)$, and $\theta_{0}$, such that $0 \leq \theta_{0}<2 \pi$, such that we have for $\nu=0, \cdots, n$, and $0<\rho<r$,

$$
\log ^{+}\left|f_{\nu}(z)\right| \leq \frac{20 R}{R-r} \sum_{\nu=0}^{n} T\left(R, f_{\nu}\right)+\left(\sum_{\nu=0}^{n} p_{\nu}\right) \log \left(\frac{R}{\rho}\right)
$$

on the path

$$
\Gamma=\Gamma\left(\theta_{0}, \rho, t\right)
$$

given by the segment

$$
\Gamma_{1}: z=\tau e^{i \theta_{0}}, \quad \rho \leq \tau \leq t
$$

followed by the circle

$$
\Gamma_{2}: z=t e^{i \theta}, \quad \theta_{0} \leq \theta \leq \theta_{0}+2 \pi
$$

Here $p_{\nu}$ is the multiplicity of the pole of $f_{\nu}$ at the origin if $f_{\nu}(0)=\infty$, and $p_{\nu}=0$ otherwise. Also in $N\left(R, f_{\nu}\right), T\left(R, f_{\nu}\right)$, we ignore poles of $f_{\nu}$ at the origin. If all the $f_{\nu}$ are analytic at the origin, we may take $\rho=0$ in $\Gamma$ and the term $\sum p_{\nu}$ in (2.1) drops out.

Our bound for the growth of the solution $y(z)$ of (1.1) follows from this and

Theorem 2.2. Suppose that $y(z)$ is analytic on an absolutely continuous path $\Gamma$ having one end point $z_{0}$ and length $l$. Suppose further that

$$
\left|y^{(\nu)}\left(z_{0}\right)\right|<K C^{\nu}, \quad 0 \leq \nu \leq n,
$$

and that for all $z$ on $\Gamma$ we have

$$
\left|y^{(n)}(z)\right|<\max \left\{K C^{n}, \sup _{0 \leq \nu \leq n-1} C^{n-\nu}\left|y^{(\nu)}(z)\right|\right\},
$$

where $K, C$ are positive constants and $n$ is a positive integer. Then we have for all $z$ on $\Gamma$ and $0 \leq \nu \leq n$,

$$
\left|y^{(\nu)}(z)\right|<K C^{\nu} e^{C l} .
$$

Combining Theorems 2.1 and 2.2 we obtain 
Theorem 2.3. Suppose that $0<\rho<r<R$ and that the path $\Gamma=\Gamma\left(\theta_{0}, \rho, t\right)$ is chosen in accordance with Theorem 2.1. We suppose that $y(z)$ is a solution of the equation (1.1) and define

$$
K=2 \max \left\{1, \sup _{0 \leq \nu \leq n}\left|y^{(\nu)}\left(z_{0}\right)\right|\right\}
$$

where $z_{0}=\rho e^{i \theta_{0}}$. We also define

$$
C=C\left(f_{\nu}, \rho, r, R\right)=(n+2) \exp \left\{\frac{20 R}{R-r} \sum_{\nu=0}^{n} T\left(R, f_{\nu}\right)+\left(\sum_{\nu=0}^{n} p_{\nu}\right) \log \left(\frac{R}{\rho}\right)\right\} .
$$

Then we have for $|z|=t$, where $t$ is some number such that $r<t<\frac{1}{4}(3 r+R)$,

$$
\left|y^{(\nu)}(z)\right|<K C^{\nu} e^{(2 \pi+1) C R}, \quad 0 \leq \nu \leq n .
$$

Proof of Theorem 2.3. In fact it follows from (1.1) and Theorem 2.1, (2.6) and (2.7) that we have on $\Gamma$

$$
\begin{aligned}
\left|y^{(n)}(z)\right| & \leq(n+1) \max \left\{\left|f_{n}(z)\right|, \sup _{0 \leq \nu \leq n-1}\left|f_{\nu}(z)\right|\left|y^{(\nu)}(z)\right|\right\} \\
& <C \max \left\{1, \sup _{0 \leq \nu \leq n-1}\left|y^{(\nu)}(z)\right|\right\} \\
& \leq \max \left\{K C^{n}, \sup _{0 \leq \nu \leq n-1} C^{n-\nu}\left|y^{(\nu)}(z)\right|\right\}
\end{aligned}
$$

since $K \geq 1$ and $C \geq 1$. Thus (2.4) holds.

Again (2.6) ensures that (2.3) holds. Thus we have (2.5). Here the length $l$ of $\Gamma$ is at most $(2 \pi+1) R$. So $(2.8)$ holds. This proves Theorem 2.3. In particular we have

$$
|y(z)|<K \exp \left\{(2 \pi+1) R(n+2) \exp \left[\frac{20 R}{R-r} \sum_{\nu=0}^{n} T\left(R, f_{\nu}\right)+\left(\sum_{\nu=0}^{n} p_{\nu}\right) \log \left(\frac{R}{\rho}\right)\right]\right\}
$$

on $\Gamma\left(\theta_{0}, \rho, t\right)$. We take logarithms on both sides and deduce that

$$
\begin{aligned}
m(t, f)< & \log K+(2 \pi+1) R(n+2) \times \\
& \times \exp \left[\frac{20 R}{R-r} \sum_{\nu=0}^{n} T\left(R, f_{\nu}\right)+\left(\sum_{\nu=0}^{n} p_{\nu}\right) \log \left(\frac{R}{\rho}\right)\right] .
\end{aligned}
$$

A blemish of Theorem 2.3 is that the estimate (2.10) is only valid for some values $t$ instead of all values $r$. This is an inevitable consequence of our method, since $y$ may have poles on $|z|=r$. If we allow for a larger bound in (2.10) then 
we can prove that the result holds for all $r$ except for some small exceptional sets. This will be done in Section 6 below.

However, since $N(r, y)$ and $T(r, y)=m(r, y)+N(r, y)$ (but not $m(r, y)$ ) are monotonic functions of $r$, we can, with the hypotheses and notation of Theorem 2.3, obtain

Theorem 2.4. We have, for $r<t<R$,

$$
T(r, y) \leq T(t, y) \leq N(t, y)+(2 \pi+1) R C+\log K
$$

The above estimate greatly improves (1.3) given by Bank and Laine [6]. If, however, $\delta=\delta(\infty, y)>0$, and $0<\varepsilon<\delta$, we have for $r$ sufficiently close to $R, N(t, y)<(1-\delta+\varepsilon / 2) T(t, y)$ and, if $T(R, y)$ is sufficiently large, $\log K<$ $\frac{1}{2} \varepsilon T(t, y)$. Thus $(2.11)$ yields

$$
T(t, y)<(1-\delta+\varepsilon) T(t, y)+(2 \pi+1) R C,
$$

This proves the following corollary.

Corollary 2.5. If $\delta=\delta(\infty, y)>0$ and $\varepsilon$ is fixed, $0 \leq \varepsilon<\delta$, we have for $r_{1}(\varepsilon)<r<R$,

$$
T(r, y) \leq\left(\frac{1}{\delta-\varepsilon}\right)(2 \pi+1) R C .
$$

We remark that if $T(t, y)$ is bounded, (2.12) still holds, since $C \rightarrow+\infty$ as $r$ approaches $R$.

The corollary indicates that we can estimate the growth of a meromorphic solution $y$, when $\delta(\infty)>0$, in terms of the Nevanlinna characteristics of the coefficients. Thus the result is sharp in view of Bank's example in (1.5) where the meromorphic solution has $\delta(\infty)=0$. This can easily be seen from [5, Lemma]. This answers a question of Milne Anderson in a private communication.

We also deduce

Corollary 2.6. If $N(t, y) / \bar{N}(t, y) \leq q$, then

$$
T(r, y) \leq(2 \pi+1) R C+\log K+q \log C .
$$

To prove Corollary 2.6 we note that the poles of $y$ must be among the poles of the coefficients $f_{\nu}$. Thus

$$
N(t, y) \leq q \bar{N}(t, y) \leq q \sum_{\nu=0}^{n} \bar{N}\left(t, f_{\nu}\right) \leq q \log C
$$

and now (2.13) follows from (2.11).

We remark that the average multiplicity $q$ must be truly enormous for the third term on the right hand side of (2.13) to dominate the first term. This is the case in the examples of Bank [5]. 
We now give some examples to test the sharpness of Theorem 2.3. We confine ourselves to the simple equation (1.5).

Example 2.7. If $f_{0}(z) \equiv 1, y(z)=a e^{z}$. Here $T\left(R, f_{0}\right)=0$,

$$
C\left(f_{0}\right)=\text { constant. }
$$

Thus we cannot dispense with the factor $R$ in (2.9).

Example 2.8. If $f_{0}(z)=z^{p}, T\left(R, f_{0}\right)=p \log ^{+} R$, then

$$
y(z)=\exp \left(\frac{z^{p+1}}{p+1}\right)
$$

and if $r=R / 2$,

$$
\log C\left(f_{0}\right)<A_{0} p \log R,
$$

where $A_{0}$ is an absolute constant. Here (2.9) yields

$$
|y(z)|<K \exp \left((2 \pi+1) R^{1+A_{0} p}\right) .
$$

This is quite sharp apart from the unknown $A_{0}$.

Example 2.9. Suppose that

$$
y(z)=\exp \exp \left(\frac{1}{1-z}\right), \quad\{z:|z|<1\} .
$$

Then

$$
f_{0}(z)=\frac{y^{\prime}(z)}{y(z)}=\frac{1}{(1-z)^{2}} \exp \left(\frac{1}{1-z}\right), \quad \text { and } \quad T\left(1, f_{0}\right)=O(1)
$$

Here

$$
C\left(f_{0}\right)=\exp \left(\frac{O(1)}{1-r}\right), \quad 0<r<1
$$

and (2.9) yields

$$
|y(z)|<\exp \exp \left(\frac{O(1)}{1-r}\right), \quad|z|=r,
$$

which again gives the right order of magnitude.

\section{Proof of Theorem 2.2}

We assume that the path $\Gamma$ is parametrized by arc length $z=z(s), 0 \leq s \leq l$, starting from the end point $z_{0}$. If we have for all $z=z(s)$ on $\Gamma$,

$$
\left|y^{(\nu)}(z)\right|<K C^{\nu} e^{C s}, \quad 0 \leq \nu \leq n
$$


then Theorem 2.2 is proved.

Suppose then that (3.1) fails for some pair $\left(z_{\nu}, \nu\right)$, when $z_{\nu}=z_{\nu}\left(s_{\nu}\right)$ and $s_{\nu}$ is chosen as small as possible. We first show that if $\nu<n$, then (3.1) also fails for $\nu+1$ and

$$
0<s_{\nu+1}<s_{\nu}
$$

In fact (2.3) ensures that (3.1) holds for $z=z_{0}$ and all $\nu$. Thus $s_{\nu}>0$. Hence if (3.2) fails, then

$$
\left|y^{(\nu+1)}(z)\right|<K C^{\nu+1} e^{C s}, \quad 0<s<s_{\nu} .
$$

Thus,

$$
\begin{aligned}
\left|y^{(\nu)}\left(z\left(s_{\nu}\right)\right)\right| & \leq\left|y^{(\nu)}\left(z_{0}\right)\right|+\left|\int_{z_{0}}^{z\left(s_{\nu}\right)} y^{(\nu+1)}(\zeta) d \zeta\right| \\
& <K C^{\nu}+K C^{\nu+1} \int_{0}^{s_{\nu}} e^{C s} d s \\
& =K C^{\nu} e^{C s_{\nu}} .
\end{aligned}
$$

Thus (3.1) holds for $z=z\left(s_{\nu}\right)$, which contradicts the definition of $s_{\nu}$ and (3.2) is proved.

We deduce that if $\nu=\nu_{0}$ is the least value of $\nu$ for which (3.1) fails, then (3.1) fails for $\nu=\nu_{0}+1, \cdots, n$, and (3.1) holds for $0 \leq \nu<\nu_{0}$, and $0 \leq s \leq l$. Also by $(3.2)$

$$
s_{n}<s_{\nu}, \quad \nu_{0} \leq \nu<n .
$$

In particular, (3.1) holds for $z=z\left(z_{n}\right)$, and for $0 \leq \nu<n$. Now using (2.4) and the definition of $s_{n}$, we obtain for $z=z\left(s_{n}\right)$,

$$
\begin{aligned}
\left|y^{(n)}(z)\right|=K C^{n} e^{C s_{n}} & <\max \left\{K C^{n}, \sup _{0 \leq \nu \leq n-1} C^{n-\nu} K C^{\nu} e^{C s_{n}}\right\} \\
& =K C^{n} e^{C s_{n}} .
\end{aligned}
$$

This contradicts the definition of $s_{n}$. Thus (3.1) and Theorem 2.2 are proved.

\section{Proof of Theorem 2.1}

To prove Theorem 2.1, we show that if $f(z)$ is meromorphic in $|z| \leq R$ then the average of $M(r, f)=\max _{|z|=r}|f(z)|$ is not too large. More precisely, we prove

Theorem 4.1. Suppose that $f(z)$ is meromorphic in $|z| \leq R$, and that $0<r<R$, $r^{\prime}=\frac{1}{4}(R+3 r)$. Then we have

$$
\frac{1}{r^{\prime}-r} \int_{r}^{r^{\prime}} \log ^{+} M(t, f) d t \leq \frac{20 R}{R-r} T(R, f)+p \log \left(\frac{R}{r}\right),
$$

where, if $f(z) \sim c z^{q}$, as $z \rightarrow 0$, we set $p=\max \{-q, 0\}$. We also define $N(R, f)=$ $N\left(R, z^{p} f\right)$ in this case; i.e., we ignore the poles of $f$ at $z=0$. 
Corollary 4.2. There exists a $t$, where $r<t<r^{\prime}$ and

$$
\log ^{+} M(t, f) \leq \frac{20 R}{R-r}\left\{T(R, f)+p \log \frac{R}{r}\right\} \text {. }
$$

A similar inequality was obtained by Nevanlinna [13, pp. 25-27], but it does not give the correct order of magnitude $1 /(R-r)$ on the right hand side of (4.2). As in the case of Nevanlinna's inequality, our estimates are also based on the Poisson-Jensen formula. See e.g. [11, p. 1].

Proof of Theorem 4.1. We need

Lemma 4.1. Suppose that $0<b<1,0<t<1,0<r<1$ and $r^{\prime}=\frac{1}{4}(1+3 r)$. We define

Then we have

$$
g(t, b)=\log \left|\frac{1-b t}{b-t}\right|
$$

$$
\frac{1}{r^{\prime}-r} \int_{r}^{r^{\prime}} g(t, b) d t<4
$$

Proof of Lemma 4.1. We adopt the main idea from the argument in [9, pp. 143145]. We use the inequality

$$
g(t, b) \leq \log ^{+}\left|\frac{1-b t}{k(b-t)}\right|+\log k
$$

where $k>1$. We note that

$$
\int_{-1}^{1} \log ^{+}\left|\frac{1}{k x}\right| d x=\int_{-1 / k}^{1 / k} \log \left|\frac{1}{k x}\right| d x=2 \int_{0}^{1 / k} \log \frac{1}{k x} d x=\frac{2}{k} .
$$

We set

$$
x=\frac{t-b}{1-b t}
$$

and assume that $|x|<1 / k$. We note that the integrand in

$$
\int_{t=0}^{t=1} \log ^{+}\left|\frac{1-b t}{k(b-t)}\right| d x \leq \frac{2}{k}
$$

vanishes if $|x| \geq 1 / k$. So it suffices to consider the range of the integration such that $|x|<1 / k$. We proceed to prove that in this case

$$
1-b<\frac{k+1}{k-1}(1-r)
$$

Suppose first that $r<1 / k$. Then the right hand side of (4.7) is greater than 1 , so that (4.7) holds. Next if $t>r \geq 1 / k$, we assume that $b<r$, since otherwise (4.7) is trivial. Then

$$
\frac{r-b}{1-b r}<\frac{t-b}{1-b t}<\frac{1}{k}
$$


since $x$ increases with $t$ in (4.5). Thus

$$
k(r-b)<1-b r, \quad b>\frac{k r-1}{k-r},
$$

and so

$$
1-b<\frac{(1+k)(1-r)}{k-r},
$$

which yields (4.7). We deduce from (4.5) and (4.7) that

$$
\begin{aligned}
d t=\frac{1-b^{2}}{(1+x b)^{2}} d x & <\frac{2(1-b)}{(1-1 / k)^{2}} d x=\frac{2 k^{2}}{(k-1)^{2}}(1-b) d x \\
& <\frac{2 k^{2}(k+1)}{(k-1)^{3}}(1-r) d x .
\end{aligned}
$$

It follows from (4.3), (4.6) and (4.8) that

$$
\begin{aligned}
\int_{r}^{r^{\prime}} g(t, b) d t & <\int_{r}^{r^{\prime}}\left(\log ^{+}\left|\frac{1-b t}{k(b-t)}\right|+\log k\right) d t \\
& <\frac{2 k^{2}(k+1)}{(k-1)^{3}}(1-r) \int_{t=0}^{t=1} \log +\left|\frac{1-b t}{k(b-t)}\right| d x+\left(r^{\prime}-r\right) \log k \\
& \leq \frac{4 k(k+1)}{(k-1)^{3}}(1-r)+\left(r^{\prime}-r\right) \log k \\
& =h(k)\left(r^{\prime}-r\right)
\end{aligned}
$$

where

$$
h(k)=\left(\frac{16 k(k+1)}{(k-1)^{3}}+\log k\right) .
$$

Thus, we aim to choose $k, k>1$, so that $h(k)$ is as small as possible. Choosing $k=22$, we obtain

$$
h(22) \approx 0.874+3.09<3.97<4 .
$$

This proves the lemma.

We can now prove Theorem 4.1. Suppose that $f(z)$ is meromorphic in $|z| \leq 1$, that $f(0) \neq \infty$, and that $0<r<1$. We apply the Poisson-Jensen formula ([11, p. 1] and [12, p. 276]) with $R=\frac{1}{4}(3+r)$ and obtain, for $z=t e^{i \theta}, r<t<r^{\prime}$,

$$
\begin{aligned}
\log |f(z)| \leq & \frac{1}{2 \pi} \int_{-\pi}^{\pi} \log \left|f\left(R e^{i \phi}\right)\right| \frac{R^{2}-t^{2}}{R^{2}-2 R t \cos (\theta-\phi)+t^{2}} d \phi \\
& +\sum_{\nu=0}^{n} \log \left|\frac{R^{2}-\overline{b_{\nu}} z}{R\left(z-b_{\nu}\right)}\right| \\
\leq & \frac{R+t}{R-t} m(R, f)+\sum_{\left|b_{\nu}\right|<R} \log \left|\frac{1-t\left|b_{\nu}\right|}{t-\left|b_{\nu}\right|}\right| .
\end{aligned}
$$


Here we have used the fact that, for fixed $z_{1}$ and $z_{2}$, the function

$$
g_{R}\left(z_{1}, z_{2}\right)=\log \left|\frac{R^{2}-\overline{z_{1}} z_{2}}{R\left(z_{1}-z_{2}\right)}\right|
$$

increases with increasing $R$ and, when $\left|z_{1}\right|,\left|z_{2}\right|$ are given, the function is maximal when $z_{1}>0, z_{2}>0$. Thus

$$
\log ^{+} M(t, f) \leq \frac{R+r^{\prime}}{R-r^{\prime}} m(R, f)+\sum g\left(t,\left|b_{\nu}\right|\right), \quad r<t<r^{\prime} .
$$

Here the sum is taken over all the $b_{\nu}$ in $\left|b_{\nu}\right|<R \leq 1$. We recall from Lemma 4.1 that $r^{\prime}=\frac{1}{4}(1+3 r)$, so that $R-r^{\prime}=\frac{1}{2}(1-r)$. Also

$$
m(R, f) \leq T(R, f) \leq T(1, f) .
$$

Thus

$$
\log ^{+} M(t, f) \leq \frac{4}{1-r} T(1, f)+\sum g\left(t,\left|b_{\nu}\right|\right) .
$$

Using Lemma 4.1, we deduce that

$$
\frac{1}{r^{\prime}-r} \int_{r}^{r^{\prime}} \log ^{+} M(t, f) d t \leq \frac{4}{1-r} T(1, f)+4 n(R, f)
$$

where $n(R, f)$ is the number of poles of $f$ in $|z|<R \leq 1$. Also

$$
T(1, f) \geq N(1, f)=\int_{0}^{1} \frac{n(t, f)}{t} d t \geq(1-R) n(R, f)=\left(\frac{1-r}{4}\right) n(R, f) .
$$

Hence

$$
\frac{1}{r^{\prime}-r} \int_{r}^{r^{\prime}} \log ^{+} M(t, f) d t \leq \frac{T(1, f)}{1-r}\{4+4 \times 4\}=\frac{20}{1-r} T(1, f) .
$$

This proves Theorem 4.1 if $R=1$, and $f(0) \neq \infty$.

Suppose next that $f$ has a pole of positive order $p$ at $z=0$. We then apply the inequality (4.9) to $F(z)=z^{p} f(z)$ instead of $f(z)$ and note that

$$
\log ^{+} M(t, f) \leq \log ^{+} M(t, F)+p \log \frac{1}{t} \leq \log ^{+} M(t, F)+p \log \frac{1}{r},
$$

and

$$
T(1, F)=T(1, f) .
$$

Thus (4.9) yields

$$
\begin{aligned}
\frac{1}{r^{\prime}-r} \int_{r}^{r^{\prime}} \log ^{+} M(t, f) d t & \leq \frac{1}{r^{\prime}-r} \int_{r}^{r^{\prime}} \log ^{+} M(t, F) d t+p \log \frac{1}{r} \\
& \leq \frac{20}{1-r} T(1, f)+p \log \frac{1}{r} .
\end{aligned}
$$


This proves Theorem 4.1 if $R=1$. In the general case we apply the above result to $f(R z)$ instead of $f(z)$ and obtain (4.1). This completes the proof of Theorem 4.1.

To complete the proof of Theorem 2.1, we need an earlier result about radial maxima. This is

Lemma 4.2. Suppose that $f$ is meromorphic in $|z| \leq R$ and that $f(0) \neq \infty$. We define

$$
f_{0}\left(r e^{i \theta}\right)=\sup _{0 \leq t \leq r}\left|f\left(t e^{i \theta}\right)\right| .
$$

Then we have for $0<r<R$,

$$
\frac{1}{2 \pi} \int_{0}^{2 \pi} \log ^{+} f_{0}\left(r e^{i \theta}\right) d \theta \leq \frac{3 R}{R-r} T(R, f) .
$$

The Lemma 4.2 is a crude version of a result $[10$, p. 183 , Theorem 1] of the second author who obtained the conclusion with

on the right hand side of (4.10) where,

$$
\left[1+\Psi\left(\frac{r}{R}\right)\right] T(R, f)
$$

$$
\Psi(t)=\frac{(1-t) \log \left(1+\frac{2 \pi \sqrt{t}}{1-t}\right)}{\pi \sqrt{t} \log (1 / t)} .
$$

We note that

$$
\log \frac{1}{t}>1-t
$$

and

$$
\log \left(1+\frac{2 \pi \sqrt{t}}{1-t}\right)<\frac{2 \pi \sqrt{t}}{1-t}
$$

Thus

$$
\Psi(t)<\frac{2}{1-t}, \quad 1+\Psi(t)<\frac{3}{1-t},
$$

and Lemma 4.2 is proved.

If $f(0)=\infty$, it is clear that $f_{0}\left(r e^{i \theta}\right)=\infty$ for $r>0$. In this case it is convenient to define

$$
f_{\rho}\left(r e^{i \theta}\right)=\sup _{\rho \leq t \leq r}\left|f\left(t e^{i \theta}\right)\right|
$$

We deduce

Lemma 4.3. Suppose that $f$ is meromorphic in $|z| \leq R$, where $0<R<+\infty$ and that $f$ has a pole of order $p$ at the origin. Then if $\rho<r<R$, we have

$$
\frac{1}{2 \pi} \int_{0}^{2 \pi} \log ^{+} f_{\rho}\left(r e^{i \theta}\right) d \theta \leq \frac{3 R}{R-r} T(R, f)+p \log \frac{R}{\rho} .
$$


Proof of Lemma 4.3. We suppose first that $R=1$ and apply Lemma 4.2 to $F(z)=$ $z^{p} f(z)$, to obtain

$$
\frac{1}{2 \pi} \int_{0}^{2 \pi} \log ^{+} F_{\rho}\left(r e^{i \theta}\right) d \theta \leq \frac{3}{1-r} T(1, F)=\frac{3}{1-r} T(1, f) .
$$

Now if $0<\rho<r<1$,

$$
f_{\rho}\left(r e^{i \theta}\right)=\sup _{\rho \leq t \leq r}\left|f\left(t e^{i \theta}\right)\right| \leq \rho^{-p} F_{\rho}\left(r e^{i \theta}\right) .
$$

Thus Lemma 4.2 yields

$$
\frac{1}{2 \pi} \int_{0}^{2 \pi} \log ^{+} f_{\rho}\left(r e^{i \theta}\right) d \theta \leq \frac{3}{1-r} T(1, f)+p \log \frac{1}{\rho} .
$$

Finally if $R \neq 1$, we apply the above conclusion to $f(R z)$ and now we obtain Lemma 4.3.

We can now complete the proof of Theorem 2.1. We choose $\rho, r$ so that $0<\rho<r<R$ and define

$$
r^{\prime}=\frac{R+3 r}{4}
$$

Then by Theorem 4.1, we have

$$
\frac{1}{r^{\prime}-r} \int_{r}^{r^{\prime}}\left(\sum_{\nu=0}^{n} \log ^{+} M\left(t, f_{\nu}\right)\right) d t \leq \frac{20 R}{R-r} \sum_{\nu=0}^{n} T\left(R, f_{\nu}\right)+\left(\sum_{\nu=0}^{n} p_{\nu}\right) \log \left(\frac{R}{r}\right)
$$

where $p_{\nu}=0$ if $f_{\nu}(0) \neq \infty$, and $p_{\nu}$ is the multiplicity of the pole of $f_{\nu}$ at $z=0$ otherwise. We deduce that there exists $t$, such that $r<t<r^{\prime}$ and

$$
\sum_{\nu=0}^{n} \log ^{+} M\left(t, f_{\nu}\right) \leq \frac{20 R}{R-r} \sum_{\nu=0}^{n} T\left(R, f_{\nu}\right)+\left(\sum_{\nu=0}^{n} p_{\nu}\right) \log \left(\frac{R}{r}\right) .
$$

In particular $\log ^{+} M\left(t, f_{\nu}\right)$ is bounded above by the right-hand side for each $\nu$. Having fixed $t$, we next choose a fixed $\rho$, with $0<\rho<r<t<R$ and deduce from Lemma 4.3 that

$$
\frac{1}{2 \pi} \int_{0}^{2 \pi} \sum_{\nu=0}^{n} \log ^{+} f_{\nu, \rho}\left(r e^{i \theta}\right) d \theta \leq \frac{3 R}{R-r} \sum_{\nu=0}^{n} T\left(R, f_{\nu}\right)+\left(\sum_{\nu=0}^{n} p_{\nu}\right) \log \left(\frac{R}{\rho}\right) .
$$

On combining this with (4.11) we deduce that on the path $\Gamma\left(\theta_{0}, \rho, t\right)$ we have for $\nu=0$ to $n$,

$$
\log ^{+}\left|f_{\nu}(z)\right| \leq \frac{20 R}{R-r} \sum_{\nu=0}^{n} T\left(R, f_{\nu}\right)+\left(\sum_{\nu=0}^{n} p_{\nu}\right) \log \left(\frac{R}{\rho}\right) .
$$

This proves Theorem 2.1. 


\section{Remarks on the unit disk case}

Bank also considers (1.5) in the unit disk in [5, Theorem 1]. In that example he constructed a solution $y(z)$ of (1.5) that can grow arbitrarily quickly as $r \rightarrow 1$ while $f_{0}(z)$ is the quotient of two bounded analytic functions in the unit disk. In particular, $f_{0}(z)$ has bounded characteristic in the unit disk ([11, p. 172]). The solution $y(z)$ constructed in this case again has the property that (1.7) grows arbitrarily quickly as $r \rightarrow 1-$.

Let $f(z)$ be a meromorphic function defined in the unit disk. Then the deficiency of $f$ can be defined in the same way as the plane:

$$
\delta_{1}(a)=1-\limsup _{r \rightarrow 1-} \frac{N(r, 1 /(f-a))}{T(r, f)},
$$

provided that $f$ has unbounded characteristic. Then the main results in Section 2 are valid in the unit disk since we may choose $R<1$ there. In particular, Theorem 2.4 asserts, as in the case of Corollary 2.5 in the plane, that the growth of a meromorphic solution of (1.1) with $\delta_{1}(\infty)>0$ can be bounded by the Nevanlinna characteristics of the coefficients of (1.1). We note that the example of Bank mentioned above has $\delta_{1}(\infty)=0$. Thus our result in the unit disk is also sharp in view of this example of Bank. We also note when the coefficients of (1.1) have bounded characteristic (for instance in (1.5)), then (2.10) shows that

$$
T(r, y)=\exp \left(\frac{O(1)}{1-r}\right),
$$

which gives the right order of magnitude as Example 2.9 shows.

\section{Estimates outside an exceptional set}

We assume that $y(z)$ is a solution meromorphic in the plane of (1.1). We write

$$
T(r)=\sum_{\nu=0}^{n} T\left(r, f_{\nu}\right),
$$

and assume that the $f_{\nu}(z)$ are not all constant so that $T(r)$ is unbounded, and more strongly

$$
T(r) \geq \log r+O(1), \quad \text { as } \quad r \rightarrow+\infty .
$$

However, we do not use the full strength of (6.2) in our first result.

We need the following growth lemma. 
Theorem 6.1. Suppose that $m(r), T(r)$ are measurable, non-negative functions for $R_{0} \leq r<+\infty$, such that $T(r)$ is non-decreasing and unbounded there and that $R_{0}>0, T\left(R_{0}\right)>1$.

We assume further that $K, \sigma$ and $p$ are constants, and that $K>0, \sigma>1$ and $p \geq 0$. For $R_{0}<r<R<+\infty$, and $r^{\prime}=\frac{1}{4}(R+3 r)$ we assume that

$$
\frac{1}{r^{\prime}-r} \int_{r}^{r^{\prime}} m(t) d t \leq \frac{K R}{R-r} T(R) .
$$

Then for $R_{0}<r<+\infty$, and outside an exceptional set $E_{p}$ that satisfies

$$
\int_{E_{p}} t^{p-1} d t<+\infty
$$

we have

$$
m(r)<T(r)\{(\log r) \log T(r)\}^{\sigma}, \quad \text { if } \quad p=0
$$

and

$$
m(r)<r^{2 p+\sigma-1} T(r)\{\log T(r)\}^{\sigma}, \quad \text { if } \quad p>0 .
$$

We note that $p=0$ corresponds to logarithmic measure and $p=1$ to Euclidean measure. Also the larger $p$ is, the smaller is the exceptional set $E_{p}$, and the weaker is the corresponding inequality (6.5) or (6.6).

We deduce

Theorem 6.2. If $y(z)$ is a solution of (1.1) which is meromorphic in the plane and the $f_{\nu}(z)$ are not all constant, we have, with the notation of Theorem 6.1, with $m(r)=m(r, y)$, and outside an exceptional set $E_{p}$ satisfying (6.4),

$$
\log m(r, y)<T(r)\{(\log r) \log T(r)\}^{\sigma}, \quad \text { if } \quad p=0 ;
$$

and

$$
\log m(r, y)<r^{2 p+\sigma-1} T(r)\{\log T(r)\}^{\sigma}, \quad \text { if } \quad p>0,
$$

where $T(r)$ is defined in (6.1), and $m(r, y)$ is the proximity function of $y$.

We first define $C_{k}=e^{k}, k=0,1,2, \cdots$. We then define a sequence $r_{\nu}, \nu=$ $1,2,3, \cdots$ inductively as follows. We set $r_{0}=R_{0}$. Suppose that $r_{\nu-1}$ has already been chosen and that

$$
C_{k} \leq T\left(r_{\nu-1}\right)<C_{k+1} .
$$

We say that $\nu$ is associated with $k$. Then we define

$$
r_{\nu}=r_{\nu-1}+k^{-\sigma} r_{\nu-1}^{1-p}
$$

We now discard as exceptional all those intervals $I_{\nu}=\left[r_{\nu-1}, r_{\nu}\right]$ for which $\nu$ is associated with $k$ and

$$
T\left(r_{\nu-1}+4 k^{-\sigma} r_{\nu-1}^{1-p}\right) \geq C_{k+1}
$$


Lemma 6.1. The union $E_{p}^{\prime}$ of the exceptional intervals satisfies

$$
\int_{E_{p}^{\prime}} t^{p-1} d t<+\infty
$$

Proof of Lemma 6.1. We show that, since $T(r)$ increases to $\infty$ with $r$, there can be, for each large $k$, at most 5 values of $\nu$ associated with $k$ and satisfying (6.9). In fact, suppose that $\nu, \cdots, \nu+q$ satisfy (6.9) and are associated with $k$, where $q \geq 5$. We deduce from (6.7) that $r_{\nu} \rightarrow+\infty$ with $\nu$ and

$$
\frac{r_{\nu}}{r_{\nu-1}}=1+k^{-\sigma} r_{\nu-1}^{-p}=(1+o(1)) \text {. }
$$

Also

$$
\begin{aligned}
r_{\nu+1}-r_{\nu}=k^{-\sigma} r_{\nu}^{1-p} & =\left(\frac{r_{\nu}}{r_{\nu-1}}\right)^{1-p}\left(r_{\nu}-r_{\nu-1}\right) \\
& =(1+o(1))\left(r_{\nu}-r_{\nu-1}\right) .
\end{aligned}
$$

Hence if $q$ is a fixed positive integer, we have

$$
r_{\nu+q+1}-r_{\nu-1}=(q+o(1))\left(r_{\nu}-r_{\nu-1}\right)=(q+o(1)) k^{-\sigma} r_{\nu-1}^{1-p} .
$$

Hence if $q \geq 5$ and (6.9) holds we deduce, if $\nu$ is sufficiently large, that

$$
T\left(r_{\nu+q-1}\right) \geq T\left(r_{\nu-1}+4 k^{-\sigma} r_{\nu-1}^{1-p}\right) \geq C_{k+1} .
$$

Thus, $T\left(r_{\nu+q-1}\right)$ does not satisfy (6.7) and so $\nu+q$ is not associated with $k$. Hence if $\nu$ is the smallest number associated with $k$ and satisfying (6.9) then the only other such numbers can be $\nu+1, \nu+2, \nu+3$ and $\nu+4$ at least if $\nu$ and $k$ are sufficiently large. Thus, if we sum over all exceptional intervals $I_{\nu}$ for which the corresponding $k$ is sufficiently large, $k \geq k_{0}$ say, and together with (6.11) and (6.12), we have

$$
\begin{aligned}
\sum_{\nu} \int_{I_{\nu}} t^{p-1} d t & \leq \sum_{\nu}(1+o(1))\left(r_{\nu}-r_{\nu-1}\right) r_{\nu-1}^{p-1} \\
& =\sum_{\nu}(1+o(1)) k^{-\sigma} \\
& \leq 6 \sum_{k \geq k_{0}}^{\infty} k^{-\sigma}<+\infty
\end{aligned}
$$

This proves Lemma 6.1.

Proof of Theorem 6.1. Suppose now that $I_{\nu}$ is a normal interval, i.e., not exceptional. In this case we set

$$
r=r_{\nu-1}, \quad R=r_{\nu-1}+4 k^{-\sigma} r_{\nu-1}^{1-p}
$$




$$
r^{\prime}=\frac{1}{4}(R+3 r)=r_{\nu-1}+k^{-\sigma} r_{\nu-1}^{1-p}=r_{\nu} .
$$

Also since $I_{\nu}$ is normal, (6.9) is false so that

$$
T(R)=T\left(r_{\nu-1}+4 k^{-\sigma} r_{\nu-1}^{1-p}\right)<C_{k+1}=e C_{k} \leq e T(r) .
$$

Thus (6.3) yields

$$
\begin{aligned}
\frac{1}{r^{\prime}-r} \int_{r}^{r^{\prime}} m(t) d t & \leq \frac{K R}{R-r} T(R) \leq \frac{e\left(r+4 k^{-\sigma} r^{1-p}\right)}{4 k^{-\sigma} r^{1-p}} K T(r) \\
& =\frac{e k^{\sigma} r^{p}\left(1+4 k^{-\sigma} r^{-p}\right)}{4} K T(r)<K k^{\sigma} r^{p} T(r),
\end{aligned}
$$

if $\nu$ and so $k$ are sufficiently large. Let $E_{\nu}$ be the subset of all those points of $I_{\nu}$ for which

$$
m(t)>\frac{K k^{\sigma} r^{p} T(r)}{\delta},
$$

where $\delta$ is a positive number. Then

$$
\left|E_{\nu}\right|<\delta\left(r^{\prime}-r\right) \text {. }
$$

Suppose first that $p=0$. In this case we choose

$$
\delta=(\log r)^{-\sigma},
$$

where $\sigma>1$. Thus, (6.14), (6.15) yield

$$
\int_{E_{\nu}} \frac{d t}{t}<\frac{1}{r}\left|E_{\nu}\right|<\frac{r^{\prime}-r}{r(\log r)^{\sigma}}<(1+o(1)) \int_{r}^{r^{\prime}} \frac{d t}{t(\log t)^{\sigma}}
$$

since $r \sim r^{\prime}$. Summing over all normal intervals we deduce that, if $\nu_{0}$ is large enough,

$$
\sum_{\nu_{0}}^{\infty} \int_{E_{\nu}} \frac{d t}{t}<2 \int_{r_{\nu_{0}}}^{\infty} \frac{d t}{t(\log t)^{\sigma}}<+\infty .
$$

On combining this with Lemma 6.1 we obtain (6.4) in this case, where $E_{p}=E_{0}$ is the union of all the exceptional intervals $I_{\nu}$ and all the subsets $E_{\nu}$ of the normal intervals $I_{\nu}$. Also, if $t$ is outside $E_{0}$ and large, then $t$ is in a normal interval $I_{\nu}$ and (6.13) is false where $\delta$ is given by (6.15). Thus

$$
\begin{aligned}
m(t) & \leq K k^{\sigma}(\log r)^{\sigma} T(r) \leq K T(r)\{(\log r) \log T(r)\}^{\sigma} \\
& =K T\left(r_{\nu-1}\right)\left\{\left(\log r_{\nu-1}\right) \log T\left(r_{\nu-1}\right)\right\}^{\sigma} \\
& \leq K T(t)\{(\log t) \log T(t)\}^{\sigma},
\end{aligned}
$$

by (6.7) and the monotonicity of $T(r)$. Also if $\sigma^{\prime}>\sigma$ we have

$$
K<(\log t)^{\sigma^{\prime}-\sigma}
$$

for large $t$ and so we have proved (6.5) with $\sigma^{\prime}$ instead of $\sigma$. Since $\sigma$ and so $\sigma^{\prime}$ can be any number greater than one we deduce that (6.5) holds. 
Suppose next that $p>0$. In this case we choose

$$
\delta=r^{1-\sigma-p} .
$$

Thus in this case (6.11) and (6.14), with $r=r_{\nu-1}$, give

$$
\int_{E_{\nu}} t^{p-1} d t<(1+o(1)) r^{p-1}\left|E_{\nu}\right|<(1+o(1)) r^{p-1} \delta\left(r^{\prime}-r\right) .
$$

On the other hand,

$$
\int_{I_{\nu}} t^{-\sigma} d t=(1+o(1)) \frac{r^{\prime}-r}{r^{\sigma}}
$$

because of (6.11). Thus (6.16) gives

$$
\int_{E_{\nu}} t^{p-1} d t<(1+o(1)) \int_{I_{\nu}} t^{-\sigma} d t .
$$

Summing over $\nu$, we deduce that, if $\nu_{0}$ is large,

$$
\sum_{\nu_{0}}^{\infty} \int_{E_{\nu}} t^{p-1} d t \leq 2 \sum_{\nu_{0}}^{\infty} \int_{I_{\nu}} t^{-\sigma} d t \leq 2 \int_{r_{\nu_{0}-1}}^{\infty} t^{-\sigma} d t<+\infty
$$

since $\sigma>1$. As in the previous case, (6.4) follows from this and Lemma 6.1. On the other hand, we have outside the exceptional set, and so in normal intervals $I_{\nu}$, where (6.13) fails,

$$
m(t) \leq \frac{K k^{\sigma} r^{p} T(r)}{\delta}=K k^{\sigma} r^{2 p+\sigma-1} T(r) \leq K t^{2 p+\sigma-1} T(t)\{\log T(t)\}^{\sigma},
$$

again by (6.7) and by the monotonicity of $T(r)$. Replacing $\sigma$ by a slightly larger number $\sigma^{\prime}$, we obtain (6.6). This proves Theorem 6.1 .

We proceed next to prove Theorem 6.2. To do this it suffices to show that

$$
m(r)=\log ^{+} m(r, y)
$$

satisfies the hypothesis (6.3) if $R_{0}$ is sufficiently large.

To do this, let $\Gamma=\Gamma\left(\theta_{0}, \rho, t\right)$ be the path whose existence is asserted in Theorem 2.1. We denote by $M_{\nu, 1}(t), M_{\nu, 2}(t), M_{\nu}(t)$ the maximum of $\left|f_{\nu}\right|$ on $\Gamma_{1}, \Gamma_{2}$ and $\Gamma$ respectively. It follows from Theorem 4.1 that

$$
\frac{1}{r^{\prime}-r} \int_{r}^{r^{\prime}} \sum_{\nu=0}^{n} \log ^{+} M_{\nu, 2}(t) d t \leq \frac{20 R}{R-r} T(R)+d_{1} \log \frac{R}{\rho},
$$

where $d_{1}$ is a constant. Similarly by Lemma 4.3 , we can choose $\theta_{0}$, depending only on $R$ so that for $t=r^{\prime}$, and so also for $\rho<t<r^{\prime}$, we have, with $r^{\prime}=\frac{1}{4}(R+3 r)$,

$$
\sum_{\nu=0}^{n} \log ^{+} M_{\nu, 1}(t) \leq \frac{3 R}{R-r^{\prime}} T(R)+d_{2} \log \frac{R}{\rho}=\frac{4 R}{R-r} T(R)+d_{2} \log \frac{R}{\rho} .
$$


Here $d_{2}$ is a constant. Thus,

$$
\frac{1}{r^{\prime}-r} \int_{r}^{r^{\prime}} \sum_{\nu=0}^{n} \log ^{+} M_{\nu, 1}(t) d t \leq \frac{4 R}{R-r} T(R)+d_{2} \log \frac{R}{\rho} .
$$

Clearly, $M_{\nu}(t)$ is the larger of $M_{\nu, 1}(t)$ and $M_{\nu, 2}(t)$ so that

$$
\begin{aligned}
\log ^{+} M_{\nu}(t) & =\max \left\{\log ^{+} M_{\nu, 1}(t), \log ^{+} M_{\nu, 2}(t)\right\} \\
& \leq \log ^{+} M_{\nu, 1}(t)+\log ^{+} M_{\nu, 2}(t) .
\end{aligned}
$$

On combining this with (6.18) and (6.19) we deduce that

$$
\frac{1}{r^{\prime}-r} \int_{r}^{r^{\prime}} \sum_{\nu=0}^{n} \log ^{+} M_{\nu}(t) d t \leq \frac{24 R}{R-r} T(R)+d_{3} \log \frac{R}{\rho},
$$

where $d_{3}=d_{1}+d_{2}$.

Let $K$ be given by (2.6). We define the constant $C$ in Theorem 2.2 by

$$
C=(n+2) \exp \left\{\sum_{\nu=0}^{n} \log ^{+} M_{\nu}(t)\right\} \text {. }
$$

Then, as in the proof of Theorem 2.3, we deduce that, when $z \in \Gamma,|y(z)|$ satisfies (2.4). Let $M(t)$ be the maximum of $|y(z)|$ on $\Gamma$, then we deduce, as in Theorem 2.3 that

$$
\log ^{+} M(t) \leq(2 \pi+1) R(n+2) \exp \left\{\sum_{\nu=0}^{n} \log ^{+} M_{\nu}(t)\right\}+K_{1} .
$$

Using (6.2) and (6.20) we deduce that for large $R$,

$$
\begin{aligned}
\frac{1}{r^{\prime}-r} \int_{r}^{r^{\prime}} \log ^{+} \log ^{+} M(t) d t & \leq \frac{24 R}{R-r} T(R)+d_{4} \log R+K_{2} \\
& \leq \frac{K R}{R-r} T(R),
\end{aligned}
$$

where $K_{1}, K_{2}, d_{4}$ and $K$ are constants. Evidently,

$$
m(t, y) \leq \log ^{+} M(t), \quad \log ^{+} m(t, y) \leq \log ^{+} \log ^{+} M(t) .
$$

Thus, we can apply Theorem 6.1 with $m(r)$ given by $(6.17)$ and the Theorem 6.2 follows from (6.5) and (6.6).

Acknowledgement. We thank the referee for a number of corrections and helpful suggestions, which have greatly improved the paper.

\section{References}

[1] S. B. Bank, A note on algebraic differential equations whose coefficients are entire functions of finite order, Ann. Scuola Norm. Sup. Pisa Cl. Sci. (3) 83 (1972), 291-197. 
[2] S. B. Bank, A general theorem concerning the growth of solutions of first-order algebraic differential equations, Compositio Math. 25 (1972), 61-70.

[3] S. B. Bank, On determining the growth meromorphic solutions of algebraic differential equations having arbitrary entire coefficients, Nagoya Math. J. 49 (1973), 53-65.

[4] S. B. Bank, On the growth of meromorphic solutions of linear differential equations having arbitrary entire coefficients, Ann. Math. Pura Appl. (4) 107 (1975), 279-289.

[5] S. B. Bank, On the existence of meromorphic solutions of differential equations having arbitrarily rapid growth, J. Reine Angew. Math. 288 (1976), 176-182.

[6] S. B. Bank and I. Laine, On the growth of meromorphic solutions of linear and algebraic differential equations, Math. Scand. 40 (1977), 119-126.

[7] M. Frei, Über die Lösungen linearer Differentialgleichungen mit ganzen Funktionen als Koeffizienten, Comment. Math. Helv. 35 (1961), 201-222.

[8] G. G. Gundersen, E. M. Steinbart and S. Wang, The possible orders of solutions of linear differential equations with polynomial coefficients, Trans. Amer. Math. Soc. 350 (1998), $1225-1247$.

[9] W. K. Hayman, The maximum modulus and valency of functions meromorphic in the unit circle, Acta. Math. 86 (1951), 89-257.

[10] W. K. Hayman, On the characteristic of functions meromorphic in the unit disk and of their integrals, Acta. Math. 112 (1964), 181-214.

[11] W. K. Hayman, Meromorphic Functions, Clarendon Press, Oxford, 1975 (with appendix).

[12] I. Laine, Nevanlinna Theory and Complex Differential Equations, W. de Gruyter, Berlin, 1993.

[13] R. Nevanlinna, Le théorème de Picard-Borel et la théorie des fonctions méromorphes, Gauthiers-Villars, Paris, 1929.

[14] Sh. Strelitz, Solutions of linear differential equations having maximal growth, J. Differential Equations 127 (1996), 542-560.

Yik-Man Chiang

Department of Mathematics

Hong Kong University

of Science and Technology

Clear Water Bay

Hong Kong

China

e-mail: machiang@ust.hk

(Received: May 2, 2002)
Walter K. Hayman

Department of Mathematics

Imperial College London

London SW7 2AZ

UK

\section{(17) To access this journal online:}

(20) http://www.birkhauser.ch 